\title{
TWO COUNTEREXAMPLES IN LOW-DIMENSIONAL LENGTH GEOMETRY
}

\author{
D. BURAGO, S. IVANOV, AND D. SHOENTHAL
}

To dear Viktor Abramovich Zalgaller on the occasion of his 85th birthday

\section{$\S 1$. INTRODUCTION}

One of the key properties of the length of a curve is its lower semicontinuity: if a sequence of curves $\gamma_{i}$ converges to a curve $\gamma$, then length $(\gamma) \leq \lim \inf \operatorname{length}\left(\gamma_{i}\right)$. Here the weakest type of pointwise convergence suffices.

There are higher-dimensional analogs of this semicontinuity for Riemannian (and even Finsler) metrics. For instance, the Besicovitch inequality (see, e.g., [1] and [4]) implies that if a sequence of Riemannian metrics $d_{i}$ on a manifold $M$ uniformly converges to a Riemannian metric $d$, then $\operatorname{Vol}(M, d) \leq \liminf \operatorname{Vol}\left(M, d_{i}\right)$. Furthermore, the same is true if the limit metric is Finsler (where any "reasonable" notion of volume for Finsler manifolds can be used); the proof, though, is more involved (see $[2,7]$ ).

However, we shall give an example of an increasing sequence of Riemannian metrics $d_{i}$ on a 2-dimensional disk $D$ that uniformly converge to a length metric $d$ on $D$ such that $\operatorname{Area}\left(D, d_{i}\right)<\frac{1}{10}$ and $\operatorname{Area}(D, d)>1$ (where by $\operatorname{Area}(D, d)$ we mean the 2-dimensional Hausdorff measure). Furthermore, the metrics $d_{i}$ and $d$ can be realized by a uniformly converging sequence of embeddings of $D$ into $\mathbb{R}^{3}$.

Our motivation for studying the semicontinuity of the surface area functional came from [3], where a more sophisticated Besicovitch-type inequality for Finsler metrics was shown. The proof is essentially Finsler, even though the inequality makes sense for general length spaces.

The counterexample undermines a natural approach to proving length-area inequalities for length spaces by means of approximations by Riemannian (more generally, Finsler) metrics. Similar considerations lead to the following question: can every intrinsic metric on a disk be approximated by an increasing sequence of Finsler metrics? There is some evidence suggesting that the answer is likely to be affirmative in dimension two. However, we shall give an example of an intrinsic metric on a 3-dimensional ball such that no neighborhood of the origin admits a Lipschitz bijection to a Euclidean region.

In this elementary exposition we present both counterexamples. Unfortunately, people often choose not to publish the results of research that led to counterexamples rather than proofs of desired theorems; as such, even published counterexamples tend to be forgotten. Hence we cannot be confident in the complete newness of the results. At the very least, we use this paper to raise open problems and embed these problems into a new context.

The paper is organized as follows. In the rest of the Introduction we give rigorous formulations of the results and outline the proofs. $\S \S 2$ and 3 contain proofs of Theorems

2000 Mathematics Subject Classification. Primary 51K10, 28A75.

Key words and phrases. Length metric, Hausdorff measure.

The second author was partially supported by RFBR (grant no. 05-01-00939a). 
1 and 2, respectively. The conclusion of $\S 4$ contains a brief discussion and several open problems.

Theorem 1. There exists a length space $(X, d)$ and a point $p \in X$ satisfying the following properties: 1) $X$ is homeomorphic to an open Euclidean ball $B \subset \mathbb{R}^{3}$;2) No neighborhood $U$ of $p$ admits a homeomorphism $\varphi: U \rightarrow V \subset \mathbb{R}^{3}$ such that $d(x, y) \geq \rho(\varphi(x), \varphi(y))$ for all $x, y \in X$, where $\rho$ is a Euclidean metric.

Note that this theorem actually gives us a length metric on a three-dimensional Euclidean ball that cannot be approximated from below by any Finsler metric. Indeed, every Finsler metric is locally bi-Lipschitz equivalent to a Euclidean one. As long as the result exists for the Euclidean metric, it will be true for any Finsler metric, since the Finsler metric is bounded by multiples of the Euclidean metric.

The proof of this first result proceeds as follows: we shall modify the standard Euclidean metric by constructing a metric as a limit of metrics that are changed on a countable collection of disjoint tori. These tori are the boundaries of tubular neighborhoods of linked circles; the circles form chains "almost" connecting two fixed points. We shall use an estimate on the distance between two linked circles in $\mathbb{R}^{3}$ via the lengths of the circles. If the metric space we construct could be mapped by a Lipschitz-1 map onto a Finsler disk, this estimate would imply that the distance between the fixed points is zero, a contradiction.

The second result is the following.

Theorem 2. The Hausdorff measure $h_{2}$ is not lower semicontinuous on length metrics on a topological disk with respect to $C^{0}$-convergence of distance functions.

A way to picture the result is to start with a sequence $\left\{d_{n}\right\}$ of length metrics defined on the same two-dimensional disk $D$. We choose the metrics $d_{n}$ so that they have a limit $d^{*}$ and so that the convergence of the length spaces $\left(D, d_{n}\right)$ to the limit space $\left(D, d^{*}\right)$ occurs in a $C^{0}$-manner. Then the limit of the two-dimensional Hausdorff measures $h_{2}\left(D, d_{n}\right)$ is strictly less than the two-dimensional Hausdorff measure $h_{2}\left(D, d^{*}\right)$.

Actually, we prove a somewhat stronger statement. We construct a sequence $\left\{D_{i}\right\}$ of embedded disks in $\mathbb{R}^{3}$ whose boundaries are all the same Euclidean circle. The result states that the $C^{0}$-limit $D_{0}$ of these disks exists and is an embedded disk in $\mathbb{R}^{3}$, and that the limit of the two-dimensional Hausdorff measures $h_{2}\left(D_{i}\right)$ of the disks $D_{i}$ is strictly less than the two-dimensional Hausdorff measure $h_{2}\left(D_{0}\right)$ of the limit disk $D_{0}$. The reader will note that, although we use elements of both interpretations, we favor the former one.

Now we outline the proof of the second theorem. We construct a surface $X$ as the union of two sets: the first is the surface of a neighborhood of an infinite tree inside the unit cube in $\mathbb{R}^{3}$, and the second is a certain Cantor set on the top face of the cube. The neighborhood of the tree thins as we approach the top of the cube. We show that this surface is homeomorphic to the standard Euclidean disk in $\mathbb{R}^{2}$. From the first point of view described above, the metrics $d_{n}$ will come from the metrics on the disk induced on the surface of certain (finite) parts of the neighborhood of the tree; the limit metric $d^{*}$ mentioned above will arise from the metric induced on the disk by the metric on the constructed surface $X$.

\section{$\S 2$. Proof of the inability to Approximate From Below By A Finsler metric}

In this section, we prove Theorem 1 . First, we shall construct the space $X$ by producing a metric on the standard Euclidean ball $B$ in $\mathbb{R}^{3}$ as a limit of metrics changed on a countable collection of disjoint solid tori.

The tori will arise as tubular neighborhoods of a certain collection of linked Euclidean circles. We shall then show that the space $X$ with the new metric $d$ is homeomorphic to 
$B$. Finally, we shall derive a contradiction if there exists a homeomorphism and a Finsler metric as in the statement of the theorem.

Construction 2.1. Now, we construct $X$. Let $a, b \in B \subset \mathbb{R}^{3}$, and choose a sequence of disjoint segments $S_{i}=\left[a_{i}, b_{i}\right]$ that lie in $B$ and converge pointwise to $[a, b]$ as $i \rightarrow \infty$ in the usual Euclidean metric.

We remark here that the metric we are constructing is not a Riemannian metric. We regard the situation as follows: consider the complement to the segment $[a, b]$. This is an open manifold, and on it we shall construct a true Riemannian metric. However, we then want to glue the segment $[a, b]$ back onto the space. What this will amount to is, in fact, compactifying the space the construction of which we have completed. We will also note that adding this key segment $[a, b]$ back into the space does not undermine the compatibility of the metrics; the metrics on the two spaces (the open manifold and $[a, b]$ ) match.

The next step is to construct a tubular neighborhood around each segment $S_{i}$. We shall alter the definition of the metric inside a portion of these tubular neighborhoods. Let us explain what will happen. For each $S_{i}$, consider a tubular neighborhood $T_{i}$ of it of radius $\epsilon_{i}$ such that:

- no two tubular neighborhoods of disjoint segments $S_{i}, S_{j}$ intersect;

- none of the tubular neighborhoods $T_{i}$ intersect $[a, b]$;

- all tubular neighborhoods are contained in $B$;

- $\epsilon_{i} \rightarrow 0$ as $i \rightarrow \infty$.

Given a segment $S_{i}$ and its tubular neighborhood $T_{i}$, we find a sequence of $n_{i}$ linked Euclidean circles $C_{i}^{j}$ contained in $T_{i}$. See Figure 1. We want the total length of all circles $C_{i}^{j}$ to be less than 10 times the Euclidean length of the segment $S_{i}$. Then, with each of the circles $C_{i}^{j}$, we associate a tubular neighborhood $T_{i}^{j}$ also contained inside $T_{i}$ such that:

- $a_{i}$ lies on the first circle $C_{i}^{1}$;

- $b_{i}$ lies on the last circle $C_{i}^{n_{i}}$;

- the radius of each tubular neighborhood $T_{i}^{j}$ is $\epsilon_{i} / 8$;

- no two distinct tubular neighborhoods $T_{i}^{j}$ and $T_{i}^{k}$ intersect.

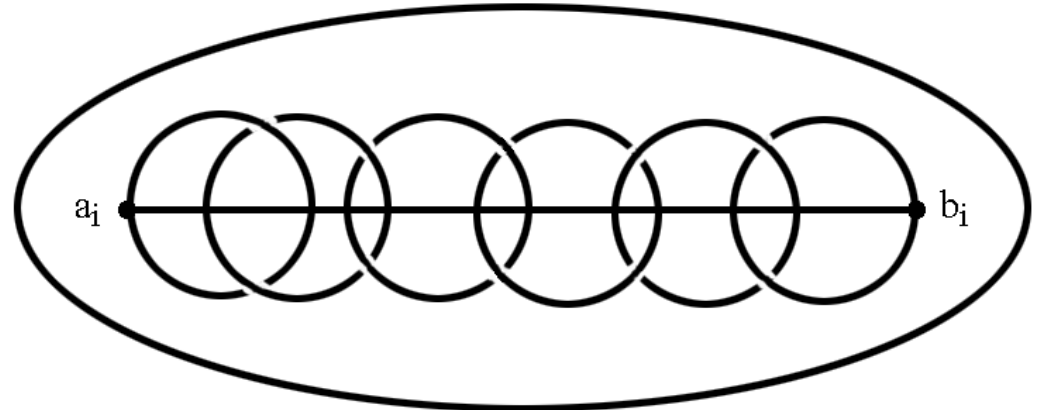

Figure 1. A sketch of the tubular neighborhood $T_{i}$.

Now we want to change the metric on the tubular neighborhood $T_{i}$. On the complement $T_{i} \backslash \bigcup_{j} T_{i}^{j}$ of the smaller tubular neighborhoods, the metric is the usual Euclidean metric. On each tubular neighborhood $T_{i}^{j}$, we change the metric as follows: first consider the smaller tubular neighborhoods $T_{i}^{j}(1) \subset T_{i}^{j}(2) \subset T_{i}^{j}(3) \subset T_{i}^{j}$ around the circles $C_{i}^{j}$ of radii $\epsilon_{i} / 32, \epsilon_{i} / 16,3 \epsilon_{i} / 32$, respectively. The plan is to make distances very small when in 
some close region of the circle $C_{i}^{j}$, but to make the price of reaching this region large by increasing the distances around it. See Figure 2 for a sketch of these changes.

To be more specific, on the tubular neighborhood $T_{i}^{j}(1)$, we multiply the Euclidean metric by a factor $1 /\left(i n_{i}\right)$. On $T_{i}^{j}(2) \backslash T_{i}^{j}(1)$, we multiply the Euclidean metric by a smooth, monotone increasing function $f$ that depends on the distance $r$ from $C_{j}^{i}$. Note that $r \in\left[\epsilon_{i} / 32, \epsilon_{i} / 16\right)$. We also require that $f\left(\epsilon_{i} / 32\right)=1 /\left(i n_{i}\right)$ and that $f\left(\epsilon_{i} / 16\right)=$ 128. On $T_{i}^{j}(3) \backslash T_{i}^{j}(2)$, we multiply the Euclidean metric by 128 . Finally, on $T_{i}^{j} \backslash$ $T_{i}^{j}(3)$, we multiply the Euclidean metric by a smooth, monotone decreasing function $g$ depending on the distance $r$ from $C_{i}^{j}$ and satisfying $g\left(3 \epsilon_{i} / 32\right)=128$ and $g\left(\epsilon_{i} / 8\right)=1$. To summarize, on each tubular neighborhood $T_{i}^{j}$, we construct a new metric by multiplying the Euclidean metric by a smooth function $h$ depending on the distance $r$ from $C_{i}^{j}$ and such that $\left.h\right|_{\left[0, \epsilon_{i} / 32\right]}=1 /\left(i n_{i}\right),\left.h\right|_{\left[\epsilon_{i} / 32, \epsilon_{i} / 16\right]}$ is monotone increasing, $\left.h\right|_{\left[\epsilon_{i} / 16,3 \epsilon_{i} / 32\right]}=128$, $\left.h\right|_{\left[3 \epsilon_{i} / 32, \epsilon_{i} / 8\right]}$ is monotone decreasing, and $h\left(\epsilon_{i} / 8\right)=1$.

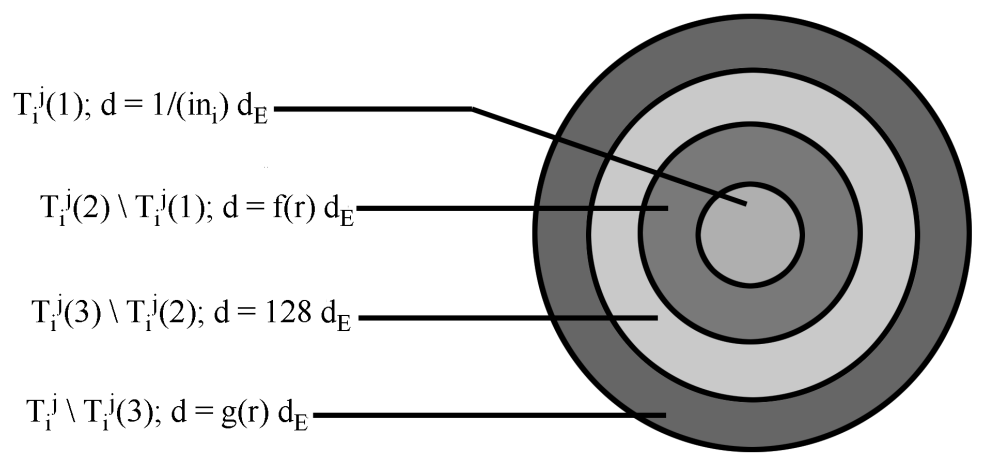

Figure 2. The change in metric.

We have defined a new metric $d$ on each tubular neighborhood $T_{i}$. On the complement of all tubular neighborhoods in $B \backslash[a, b]$, let $d$ be the usual Euclidean metric. In this way, we have defined a new metric $d$ on all of $B \backslash[a, b]$.

The final step is to glue the segment $[a, b]$ back onto our manifold. This gluing completes the construction of the space $X$.

We note that gluing the segment $[a, b]$ back onto the manifold is the same as compactifying the manifold. To demonstrate this fact in quite specific terms, we need the following statement.

Lemma 2.2. For any two points $p, q \in[a, b] \subset(X, d)$, the segment $[p, q] \subset[a, b]$ remains the shortest path between $p$ and $q$.

Proof. To begin with, we note an important property of $d$ :

- On any given tubular neighborhood $T_{i}, d$ is sandwiched between $1 /\left(i n_{i}\right) d_{E}$ from below and $128 d_{E}$ from above.

With this property in mind, we estimate the length of a segment going through a tubular neighborhood $T_{i}^{j}$. The segment through $T_{i}^{j}$ which intersects $C_{i}^{j}$ has length

$$
2 \int_{0}^{\epsilon_{i} / 8} h(x) d x \geq 2 \int_{0}^{\epsilon_{i} / 32} \frac{1}{i n_{i}} d x+2 \int_{\epsilon_{i} / 16}^{3 \epsilon_{i} / 32} 128 d x=\frac{\epsilon_{i}}{16 i n_{i}}+8 \epsilon_{i} .
$$

Thus, any shortest path (with respect to the Euclidean distance) that originally passed through the smaller tubular neighborhood $T_{i}^{j}(1)$ will be shorter in the metric $d$. However, any shortest path with respect to the Euclidean distance that originally passed 
through every smaller tubular neighborhood of $T_{i}^{j}$ will see its length increase drastically. Also, given two points, if the shortest path between them with respect to the Euclidean distance did not pass through every smaller tubular neighborhood of $T_{i}^{j}$, then the shortest path between them with respect to $d$ would not pass through every smaller tubular neighborhood of $T_{i}^{j}$ either.

Notice, then, that the shortest path between points $p, q$ on the segment $[a, b]$ under the metric $d$ will be along $[a, b]$.

We have been stating without proof that $d$ is a metric on the space $X$. We now prove this formally (note that $d$ is not bounded away from zero).

Lemma 2.3. $d$ is a metric on $X$.

Proof. To show that $d$ is a metric, we prove that the distances under $d$ are finite and that no distinct points are identified as having zero distance with respect to $d$.

To see that the $d$-distances are finite, it suffices to observe that the segment between two points still has finite distance under $d$. Therefore, the $d$-distance between any two points is less than or equal to the length of the segment between those two points under $d$. The length of this segment under $d$ is at most 128 times its Euclidean length, and thus, the $d$-distances are finite.

Now we want to show that if $d(p, q)=0$, then $p=q$. First, notice that we did not change the metric on $[a, b] \cup\left(\cup T_{i}\right)^{c}$. Thus, if both $p$ and $q$ are in $[a, b] \cup\left(\cup T_{i}\right)^{c}$, then there are neighborhoods of $p$ and $q$ in which the shortest path is a segment. This segment must have nonzero length in these neighborhoods, and so $d(p, q) \neq 0$ unless $p=q$.

If $p$ and $q$ are in $\bigcup T_{i}$, let $p \in T_{j}, q \in T_{k}$, where both metrics are finite. At worst, $p, q$ are both in a tubular neighborhood $T_{j}^{l}(m)$ for $m=1,2$. But if this is true, the distance between $p$ and $q$ is bounded from below by $1 /\left(j n_{j}\right) d_{E}(p, q)$.

The final case to consider is that of $p \in[a, b], q \in \bigcup T_{i}$. But again, let $q \in T_{j}$, and then $d(p, q) \geq d_{E}(p, q)$ as above.

Our next step is to show that the topology of $B$ is unchanged under the change in metric.

Lemma 2.4. A sequence $\left\{x_{n}\right\}$ in $B$ converges under the metric $d$ if and only if it converges under the Euclidean metric $d_{E}$.

Proof. First, assume that a sequence $\left\{x_{n}\right\}$ converges to a point $x$ with respect to the metric $d$. We must show that $\left\{x_{n}\right\}$ converges to $x$ with respect to the Euclidean metric $d_{E}$. There are two cases. If $x \in T_{i}^{j}(m)$ for $m=1,2$ and some $i, j$, then also $x_{n} \in T_{i}^{j}(m)$ for $m=1,2$ if $n$ is sufficiently large. In this case, $d\left(x_{n}, x\right) \geq 1 /\left(i n_{i}\right) d_{E}\left(x_{n}, x\right)$. So, $d\left(x_{n}, x\right) \rightarrow 0$ implies $1 /\left(i n_{i}\right) d_{E}\left(x_{n}, x\right) \rightarrow 0$, which in turn means that $d_{E}\left(x_{n}, x\right) \rightarrow 0$. Thus, $\left\{x_{n}\right\}$ converges to $x$ in $d_{E}$.

On the other hand, if we assume that $x$ is not in some $T_{i}^{j}(m)$ for $m=1,2$, then $d\left(x_{n}, x\right) \geq d_{E}\left(x_{n}, x\right)$. Thus, if the sequence converges in the former metric, it converges in the latter.

Now we must show that if a sequence $\left\{x_{n}\right\}$ converges to $x$ with respect to $d_{E}$, then the sequence converges to the same point in $(B, d)$. Since $d$ is bounded from above by $128 d_{E}$, the relation $d_{E}\left(x_{n}, x\right) \rightarrow 0$ implies $d\left(x_{n}, x\right) \rightarrow 0$, as required.

The preceding lemmas show that $(X, d)$ is homeomorphic to $\left(B, d_{E}\right)$. Thus, we have proved the first property in Theorem 1 for this particular ball.

To prove the second property for this ball $B$, we must show that one cannot choose both a (Finsler) metric $\rho$ on $B$ and a homeomorphism $\varphi: X \rightarrow B$ as in the theorem. We argue by contradiction and assume that both exist. To find a contradiction, we require 
another lemma, which estimates the distance between linked curves in terms of their lengths.

Lemma 2.5. There is a constant $C$ such that for any Finsler metric $\rho$, there is an $\epsilon>0$ such that, given two linked curves $\gamma_{1}, \gamma_{2}$ of length less than $\epsilon$, we have $\rho\left(\gamma_{1}, \gamma_{2}\right) \leq$ $C \min \left(l_{\rho}\left(\gamma_{1}\right), l_{\rho}\left(\gamma_{2}\right)\right)$.

Proof. First, we reduce the problem to looking at constants associated with a Euclidean metric. Then we shall show that finding a constant in that case is straightforward. So, given a Finsler metric $\rho$, choose $\epsilon>0$ such that every $100 \epsilon$-ball admits a 10 bi-Lipschitz homeomorphism to a Euclidean region. Having done this, we can concentrate on the case of a Euclidean metric. Let $\gamma_{1}, \gamma_{2}$ be two linked curves, the lengths of which are less than $\epsilon$. Assume without loss of generality that $\gamma_{1}$ is the shorter of the two curves. Thus, $\gamma_{2}$ intersects any immersed disk whose boundary is $\gamma_{1}$. Choose a point $x$ on $\gamma_{1}$ and consider the immersed disk $D$ defined by connecting every point of $\gamma_{1}$ to $x$ by a shortest path. Each of these shortest paths must have length less than or equal to $\left|\gamma_{1}\right| / 2$. Since $\gamma_{2}$ intersects this immersed disk $D$ (and hence, one of the segments $\left[x, \gamma_{1}(t)\right]$ ), it must be within $\left|\gamma_{1}\right| / 4$ of the boundary $\partial D=\gamma_{1}$. So, we have shown in this case that the constant is $1 / 4$. The constant for the Finsler metric $\rho$ is at most $10 / 4$.

With this lemma at hand, we can complete our result for this ball.

Lemma 2.6. A (Finsler) metric $\rho$ on $B$ and a homeomorphism $\varphi: X \rightarrow B$ such that $d(x, y) \geq \rho(\varphi(x), \varphi(y))$ for all $x, y \in X$ cannot exist simultaneously.

Proof. Assume that, to the contrary, both a metric $\rho$ on $B$ and a homeomorphism $\varphi$ as above exist. As has already been mentioned, we shall need Lemma 2.5 to arrive at a contradiction. So, if $\rho$ exists and we are able to show that $d\left(a_{i}, b_{i}\right) \leq \sum d\left(C_{k}^{i}, C_{k+1}^{i}\right)$, the required contradiction arises.

First, note that the length of the circle $C_{i}^{j}$ in the metric $d$ is $2 \pi /\left(i n_{i}\right)$. If the above assumption is true, the lengths of the curves are less than a given $\epsilon$ for $i$ sufficiently large. Thus,

$$
\rho\left(\varphi\left(a_{i}\right), \varphi\left(b_{i}\right)\right) \leq d\left(a_{i}, b_{i}\right) \leq \sum_{k=1}^{n_{i}} l\left(C_{k}^{i}\right)+\sum_{k=1}^{n_{i}-1} d\left(C_{k}^{i}, C_{k+1}^{i}\right) \leq 2 \pi\left(\frac{1}{i}+\left(n_{i}-1\right) C \frac{1}{i n_{i}}\right),
$$

which goes to zero as $i$ goes to infinity. But this implies that $\rho(\varphi(a), \varphi(b))=0$, which is impossible because $\varphi$ is a homeomorphism.

To prove Theorem 1 in full generality, fix a point $p \in B$ and repeat the described procedure on a sequence of disjoint balls whose radii tend to zero and the balls themselves converge to $p$. Since the result is true on each ball in the sequence, it is true for any neighborhood of $p$.

\section{§3. Hausdorff measure on Length metrics on a topological Disk FAILS TO BE LOWER SEMICONTINUOUS}

In this section, we prove Theorem 2. To give an example of a convergent sequence of spaces with the property that the limit of their 2-dimensional Hausdorff measures is strictly less than the 2-dimensional Hausdorff measure of the limit space, we first construct an infinite tree in the unit cube in $\mathbb{R}^{3}$ whose branches tend to the top face of the cube. The surface we construct will include the surface of a certain neighborhood of the tree; the neighborhood will thin as we travel "up" the tree. Also included in the surface will be a Cantor set in the top face of the cube. Proceeding with the proof, we 
shall show that this surface is homeomorphic to the standard 2-disk in $\mathbb{R}^{2}$. Then, our sequence of spaces will arise from each step of the tree, and we shall show the required claim.

Construction 3.1. In order to determine the $x$ and $y$ coordinates of the points on the tree, consider the following Cantor set construction. At step 0, we have the unit interval. At step 1, we remove the center interval of length $1 / 4$ (so we have $[0,3 / 8] \cup[5 / 8,1]$ ). At step $k$ of the procedure, from the remaining subintervals we remove the $2^{k-1}$ center intervals of equal length and such that the total length of the intervals removed on that step is $1 / 2^{k+1}$. In this way, the resulting Cantor set will have positive Lebesgue measure. With each of the $2^{k}$ endpoints of the intervals removed at step $k$, we associate the two endpoints of the subinterval removed at step $k+1$ that lie between an endpoint of step $k$ and the closest endpoint of step $k-1$. We also introduce the notation $c_{k}^{i}$ to mean the center of the $i$ th interval removed at step $k$.

Now, we construct the tree. As the initial vertex, we choose the point $(1 / 2,1 / 2,0)$. From this vertex, we extend 4 edges, one to each point $(x, y, 3 / 4)$, where $x, y \in\{3 / 8,5 / 8\}$. Then we proceed by induction. More specifically, at step $k$, given a point of the tree $\left(x, y, 1-1 / 4^{k}\right)$ (where each of $x$ and $y$ is an endpoint of a subinterval removed at step $k$ of the Cantor set construction described above), we add 4 edges linking this point with $\left(x_{1,2}, y_{1,2}, 1-1 / 4^{k+1}\right)$, where the $x_{1,2}$ are the two points associated with $x$ as described in the preceding paragraph, and the $y_{1,2}$ are the two points associated with $y$. Thus, every step- $k$ point gives rise to 4 new points and, accordingly, to 4 new edges. In this way, we have constructed a tree $T$ in the unit cube.

Now we use this tree as a skeleton around which we build a body - a topological surface $X$. First, around the tree we construct a tube-like neighborhood. Fix a positive $\epsilon<1$. Around each vertex at height $1-1 / 4^{k}$, we consider the Euclidean ball of radius $\epsilon / 4^{k+2}$. Around each edge that connects vertices at heights $1-1 / 4^{k}$ and $1-1 / 4^{k+1}$, we consider the tubular neighborhood of radius $\epsilon / 4^{k+3}$. Note that the choice of radii of the tubular neighborhoods prevents the intersection of tubular neighborhoods around distinct edges. In regions where the tubular neighborhood around a vertex and tubular neighborhood around an edge intersect, we adjust the neighborhoods so that they meet smoothly. We also replace the ball around the first vertex (the one at height 0 ) by a halfsphere. This is needed to keep our space within the unit cube. It is also because of this replacement, and the fact that tubular neighborhoods cannot thin as we have described, that we sacrifice the term "tubular neighborhood" in favor of "tube-like neighborhood".

Because of the inductive nature of the construction of the tree, we shall view the construction of the topological surface $X$ as a step-by-step process by labeling each successive tube-like neighborhood. Let $X_{0}$ be the half-sphere of radius $1 / 16$ centered at the point $(1 / 2,1 / 2,0)$ in the unit cube. $X_{1}$ is the space determined by the (boundary of the) tube-like neighborhood of the (partial) tree consisting of 5 vertices ( 1 vertex at height 0 and 4 vertices at height $3 / 4$ ) and the edges connecting them. Specifically, to form $X_{1}$, we consider the addition of what we shall call capped neighborhoods to $X_{0}$. Each capped neighborhood is a tube-like neighborhood around an edge "capped" by a sphere around the vertex at the end of the edge with higher $z$-coordinate. See Figure 3 for an illustration.

We proceed inductively. Given the space $X_{n}$, we define the space $X_{n+1}$ as the union of $X_{n}$, the tube-like neighborhoods around all vertices at height $1-1 / 4^{n+1}$, and the set of tube-like neighborhoods around all edges connecting those vertices to points of $X_{n}$. In other words, $X_{n+1}$ is the union of $X_{n}$ and the $4^{n+1}$ capped neighborhoods emanating from $X_{n}$. In essence, any space $X_{n}$ consists of pieces of spheres and cylinders glued together in a particular way. 


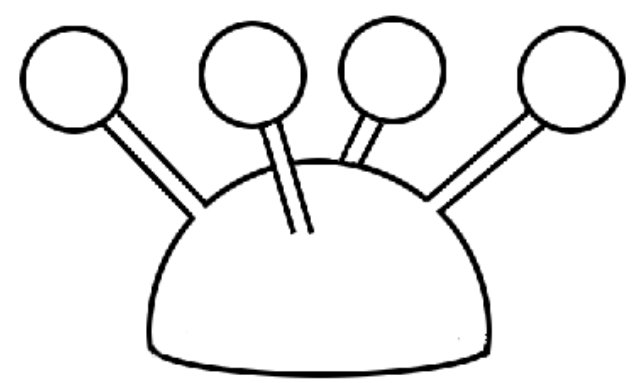

FiguRE 3. A sketch of the space $X_{1}$.

The definition of the space $X$ is completed by taking the closure of the union of the spaces $X_{n}$ above. The limit points we add are at height 1 , and we shall associate them with points of a certain Cantor set in the disk $D$.

Now the construction of $X$ is complete, and we show that $X$ is homeomorphic to the two-dimensional disk $D$. We proceed in two steps. First, we define inductively a sequence of maps (which will in fact be homeomorphisms) between the spaces $X_{i}$ and the two-dimensional unit disk. Then we show that this limit of this sequence is itself a homeomorphism.

Lemma 3.2. Each space $X_{i}$ is homeomorphic to the two-disk D.

Proof. To begin with, we map $X_{0}$ (recall that this is the half-sphere of radius $1 / 16$ centered at the point $(1 / 2,1 / 2,0)$ of the unit cube) to the standard unit disk $D$ in $\mathbb{R}^{2}$ via a homeomorphism $F_{0}$. To define the map $F_{1}$, consider the unit disk $D$ as divided into five regions: four are smaller disks of radius $1 / 4$ about the points $( \pm 1 / \sqrt{8}, \pm 1 / \sqrt{8})$, and the fifth region is the complement of those four disks in the disk $D$. On that complement, let $F_{1}=F_{0}$. On each of the four smaller disks, define $F_{1}$ to be a homeomorphism between one of the four capped tube-like neighborhoods of $X_{1}$ and one of the four smaller disks defined previously. We require that $F_{1}$ be smooth at the boundary of the smaller disks. We proceed by induction. Given a homeomorphism $F_{n}$ between the space $X_{n}$ and $D$, we define a homeomorphism $F_{n+1}: X_{n+1} \rightarrow D$ as follows. Consider the smallest $4^{n}$ disks of radius $1 / 4^{n}$ on which $F_{n} \neq F_{n-1}$. We view each of these disks $\bar{D}$ as consisting of five parts: four smaller disks of radius $1 / 4^{n+1}$ centered at points that are at a distance $1 / 2^{2 n+1}$ from the center of $\bar{D}$ and the complement of those smaller disks. On that complement, we let $F_{n+1}=F_{n}$. Otherwise, we define $F_{n+1}$ to be a homeomorphism between one of the capped neighborhoods (whose cap has center at height $1-1 / 4^{n+1}$ ) and one of the four smaller disks. We again require that $F_{n+1}$ be smooth on the boundary of the four smaller disks.

Now, we define a map $F$ between the space $X$ and $D$ as the limit of the above homeomorphisms $F_{n}$.

Lemma 3.3. $F: X \rightarrow D$ is a homeomorphism.

Proof. Since $F$ is defined between compact spaces, we need only show that it is injective and continuous.

To show that $F$ is injective, choose two points $x_{1} \neq x_{2} \in X$. If $x_{1}$ or $x_{2}$ is in the union $\bigcup_{n} X_{n}$, assume without loss of generality that $x_{1} \in X_{k}$. Then $F=F_{k}$ in a 
small neighborhood of $x_{1}$, in which case it is a homeomorphism (and hence, injective). Now, assume that $x_{1}$ and $x_{2}$ are distinct points in $X \backslash\left(\bigcup_{n} X_{n}\right)$. Fix two sequences $\left\{x_{1}^{n}\right\}$ and $\left\{x_{2}^{n}\right\}$ converging to $x_{1}$ and $x_{2}$, respectively. For any $n$, consider the capped neighborhoods in $X_{n} \backslash X_{n-1}$. The sequence $\left\{x_{1}^{n}\right\}$ must eventually remain in one of (the extensions of) those specific capped neighborhoods; we denote this (extension of a) capped neighborhood by $X_{n}^{1}$. A similar situation occurs for $\left\{x_{2}^{n}\right\}$, yielding $X_{n}^{2}$. Since $x_{1}$ and $x_{2}$ are distinct, for some $N$ we have $X_{N}^{1} \neq X_{N}^{2}$, and thus the images of the sequence under the maps $F_{k}$ will always be a given distance apart. This implies that the distance between $F\left(x_{1}\right)=\lim _{n} F\left(x_{1}^{n}\right)$ and $F\left(x_{2}\right)=\lim _{n} F\left(x_{2}^{n}\right)$ must be nonzero. Thus, $F$ is injective.

To show that $F$ is continuous, we consider a sequence $\left\{x_{n}\right\}$ in $X$ that converges to a point $x$. We want to demonstrate that the sequence $\left\{F\left(x_{n}\right)\right\}$ converges to $F(x)$. If $x \in \bigcup_{n} X_{n}$, then $x$ is in $X_{k}$ for some $k$. Hence, $\left\{x_{n}\right\}$ must eventually be in $X_{k+1}$. Therefore, $F\left(x_{n}\right)=F_{k+1}\left(x_{n}\right)$ for sufficiently large $n$, and so the sequence of images must converge to $F(x)$. If $x \in X \backslash\left(\bigcup_{n} X_{n}\right)$, then with $x$ we can associate a word from the language with 4 symbols. We proceed by induction. We start with associating the values of $1,2,3$, or 4 with the capped neighborhoods in $X_{1}$ in accordance with the quadrant the projection of the cap lies in (we use the Cartesian coordinate system whose origin is $(1 / 2,1 / 2))$. Then, if a finite sequence $A_{1} A_{2} \ldots A_{N}$ has already been assigned to a capped neighborhood $C$ in $X_{N}\left(A_{i} \in\{1,2,3,4\}\right)$, we can then assign a sequence $A_{1} A_{2} \ldots A_{N} A_{N+1}$ to each of the four capped neighborhoods stemming from $C$ in $X_{N+1}$. The value $A_{N+1} \in\{1,2,3,4\}$ is determined by what quadrant the projection of the cap from the new capped neighborhood is in, by using the Cartesian coordinate system whose origin is determined as follows: if the center of the cap is $\left(x, y, 1-1 / 4^{N}\right)$, where $x$ is an endpoint of the $i$ th subinterval and $y$ is an endpoint of the $j$ th subinterval at step $N$, then the origin is found at $\left(c_{N}^{i}, c_{N}^{j}\right)$. Now, notice that the points in $D$ that are not in the image of any $F_{n}$ can also be numbered in a similar way. Indeed, $F$ is seen to map points in $X \backslash\left(\bigcup_{n} X_{n}\right)$ with associated word $A_{1} A_{2} \ldots$ to points in $D$ with the same associated word. Therefore, the images of convergent sequences in $X$ converge in $D$ to the image of the limit.

Since $F$ is a homeomorphism (as well as all the $F_{n}$ ), we endow $X$ and each $X_{n}$ with the metric induced from $D$ by the associated homeomorphism. That is, the metric $d^{*}$ on $X$ is defined by $d^{*}(x, y)=d_{E}(F(x), F(y))$, where, as usual, $d_{E}$ is the standard Euclidean metric. Similarly, we have metrics $d_{n}$ on $X_{n}$ induced by the homeomorphisms $F_{n}: X_{n} \rightarrow D$.

In order to refer to all $X_{n}$ and $X$ as $D$, we need to show that the intrinsic metric $d$ induced from the ambient space determines the same topology on $X$ or $X_{n}$ as the intrinsic metric $d^{*}$ or $d_{n}$, respectively. It will suffice to show the equivalence under $d$ and $d^{*}$, and, in fact, by using only points $x, y \in X \backslash\left(\bigcup_{n} X_{n}\right)$. The arguments for at least one point in $\bigcup_{n} X_{n}$ are similar.

Let $x, y$ be two distinct points in $X \backslash\left(\bigcup_{n} X_{n}\right)$. We want to find constants $C_{1}$ and $C_{2}$ such that $d(x, y) \leq C_{1} d^{*}(x, y)$ and $d^{*}(x, y) \leq C_{2} d(x, y)$. Let $x=A_{1} A_{2} \ldots A_{N} \ldots$ and $y=B_{1} B_{2} \ldots B_{N} \ldots$, where $A_{i}, B_{i} \in\{1,2,3,4\}$ as in the proof of the continuity of $F$. In addition, let $N$ be the number such that $A_{N} \neq B_{N}$ but $A_{i}=B_{i}$ for all $i<N$. Then the distance between $x$ and $y$ in the ambient metric is bounded above by twice the sum of the lengths of certain halves of great circles on spheres at each of the heights $1-1 / 4^{N}$ upward plus the sum of the lengths of the segments between the centers of those spheres at each height. It can be shown that this sum is strictly less than $4 / 4^{N}$. However, the distance in the induced metric $d^{*}(x, y)$ between the points $x$ and $y$ is at least the distance between two circles of radius $1 / 4^{N+1}$ as detailed in the construction of 
the homeomorphisms $F_{n}$. A calculation yields $d^{*}(x, y) \geq\left(1 / 4^{N}\right) / 5$. We conclude that $d(x, y) \leq 20 d^{*}(x, y)$.

Now we must find the constant $C_{2}$. Since $x$ and $y$ have the same representation in 4 digits until the $N$ th term, their images under the homeomorphism $F$ can be no more than $1 / 4^{N}$ apart. However, the distance between $x$ and $y$ in $X$ is certainly not smaller than twice the length of a segment from $x$ to the center of the sphere at the height where they separate. Namely, $d(x, y) \geq 2 / 4^{N}$. Thus, $d^{*}(x, y) \leq 2 d(x, y)$, and we see that the topologies are equivalent under the metrics $d$ and $d^{*}$. Since all $X_{n}$ and $X$ are homeomorphic to $D$, we now refer to them as $D$.

To complete the proof of Theorem 2, we need the final lemma.

Lemma 3.4. The limit of the two-dimensional Hausdorff measures of $\left(D, d_{n}\right)$ is strictly less than the two-dimensional Hausdorff measure of $\left(D, d^{*}\right)$.

Proof. Recall that the radius of the tubes was a factor of some chosen $\epsilon$. Therefore, the two-dimensional Hausdorff measure of the spaces $\left(D, d_{n}\right)$ can be made as small as we wish by reducing the value of $\epsilon$. On the other hand, the two-dimensional Hausdorff measure of $\left(D, d^{*}\right)$ is bounded from below by the (strictly positive) Hausdorff measure of the Cantor set consisting of the points of $X \backslash\left(\bigcup_{n} X_{n}\right)$. Hence, with the proper choice of $\epsilon$, we have $\lim _{n} h_{2}\left(D, d_{n}\right)<h_{2}\left(D, d^{*}\right)$, and the failure of lower semicontinuity of Hausdorff measure on topological disks is shown.

\section{§4. DisCUSSION OF OPEN PROBLEMS}

In this concluding section, we would like to discuss several open problems and directions for further research with regard to the two questions that began our paper. These questions are related to problems posed in $[5,6]$ and [3].

In two dimensions, it seems likely that every length metric is the limit of an increasing sequence of Finsler metrics. The following fact serves as supporting evidence: every two-dimensional disk with a length metric admits a Lipschitz-1 surjection to a Euclidean region. So far, we cannot rule out the situation when this surjection collapses some (connected) parts of the disk to singletons, but we have a feeling that this can be fixed.

In higher dimensions, the situation is less clear. One possible question to ask is as follows: what "reasonable" restrictions (if any) can be placed on a class of sequences of Finsler metrics such that all manifolds with length metrics can be obtained as limits of those sequences, and the volume (i.e., Hausdorff measure) is lower semicontinuous?

With regard to the second question, we remark that it is true in any dimension that Hausdorff measure is lower semicontinuous with respect to uniform convergence if all metrics in question (including the limit metric) are Riemannian. Furthermore, the same is true if the limit metric is Finsler; however, the proof is more involved (see [7]). In two dimensions, the lower semicontinuity of the symplectic Finsler surface area (also known as the Holmes-Thompson area; see [8]) is known for Finsler metrics [3]. However, it is interesting to note that, at this point, the same statement has no answer even in two dimensions if we keep the assumptions of Finsler elements in the sequence and a smooth, Finsler limit metric but consider Hausdorff measure instead.

\section{REFERENCES}

[1] A. Besicovitch, On two problems of Loewner, J. London Math. Soc. 27 (1952), 141-144. MR0047126 $(13: 831 d)$

[2] D. Burago and S. Ivanov, On asymptotic volume of tori, Geom. Funct. Anal. 5 (1995), 800-808. MR1354290 (96h:53041)

[3] _ On asymptotic volume of Finsler tori, minimal surfaces in normed spaces, and symplectic filling volume, Ann. of Math. (2) 156 (2002), 891-914. MR1954238 (2003k:53088) 
[4] Yu. D. Burago and V. A. Zalgaller, Geometric inequalities, "Nauka", Leningrad, 1980; English transl., Grundlehren Math. Wiss., Bd. 285, Springer-Verlag, Berlin, 1988 . MR0936419 (89b:52020)

[5] H. Busemann, Intrinsic area, Ann. of Math. (2) 48 (1947), 234-267. MR0020626 (8:573a)

[6] H. Busemann, G. Ewald, and G. Shephard, Convex bodies and convexity on Grassmann cones. I-IV, Math. Ann. 151 (1963), 1-41. MR0157286 (28:522a)

[7] S. Ivanov, Gromov-Hausdorff convergence and volumes of manifolds, Algebra i Analiz 9 (1997), no. 5, 65-83; English transl., St. Petersburg Math. J. 9 (1998), no. 5, 945-959. MR1604389 (98k:53052)

[8] A. C. Thompson, Minkowski geometry, Encyclopedia Math. Appl., vol. 63, Cambridge Univ. Press, Cambridge, 1996. MR1406315 (97f: 52001)

Department of Mathematics, The Pennsylvania State University, University Park, PennSYLVANIA 16802

E-mail address: burago@math.psu.edu

St. Petersburg Branch, Steklov Mathematical Institute, Russian Academy of Sciences, Fontanka 27, St. Petersburg 191023, Russia

E-mail address: svivanov@pdmi.ras.ru

Longwood University, 201 High Street, Farmville, Virginia 23909

Received 15/FEB/2006

Originally published in English 